\title{
Fauna edáfica influenciada pelo uso de culturas e consórcios de cobertura do solo ${ }^{1}$
}

\author{
Rodrigo Ferreira da Silva², Geomar Mateus Corassa², \\ Gilvan Moisés Bertollo ${ }^{2}$, Antonio Luis Santi ${ }^{2}$, Ricardo Bemfica Steffen ${ }^{3}$
}

\begin{abstract}
Edaphic fauna influenced by the use of soil cover crops and intercropping

The edaphic fauna is considered sensitive and responds relatively fast to the impact of different types of production systems, thereby enabling its use as an active component for evaluating soil quality. This study was conducted to determine the influence of black oat, turnip, vetch and the intercropping of turnip/black oat and vetch/turnip/black oat in the structure and composition of the edaphic fauna. The experimental design was randomized blocks, in a $6 \times 4$ factorial scheme, consisting of six soil cover crops (black oat, turnip and vetch, the intercropping of turnip/black oat and vetch/turnip/black oat and fallow ground) and four sampling times $(0,50,100$ and 150 days after sowing), with four replications. After collecting the soil fauna, the abundance of individuals, Collembola number, Margalef richness, Shannon diversity index, Pielou equitability and Simpson dominance were determined. The intercropping of turnip/black oat and vetch/turnip/ black oat favored the abundance of individuals and the Collembola number, within the first 50 days. The intercropping of vetch/turnip/ black oat reduced the Shannon diversity index by increasing the Collembola population. The highest species diversity was observed at 100 days after the crops implantation. The edphic fauna was influenced by the vegetative stage and soil cover crop types.
\end{abstract}

KEY-WORDS: Avena stringosa; Raphanus sativus L.; Vicia sativa; ecological indexes; bioindicators.

\section{INTRODUÇÃO}

Plantas de cobertura favorecem a ciclagem de nutrientes, a agregação, o armazenamento de água e a manutenção da matéria orgânica, em comparação com os monocultivos anuais (Alcântara et al. 2000, Boer et al. 2007). Algumas espécies, devido à decomposição dos seus resíduos, podem promover consideráveis aumentos de rendimento nas culturas subsequentes (Costa et al. 2011). Neste sentido,

\section{RESUMO}

A fauna edáfica é sensível e responde, com relativa rapidez, ao impacto de diferentes tipos de sistemas de produção, possibilitando, deste modo, o seu uso como componente ativo na avaliação da qualidade do solo. O presente trabalho objetivou determinar a influência do cultivo de aveia preta, nabo e ervilhaca e dos consórcios nabo/aveia preta e ervilhaca/nabo/aveia preta, na estrutura e composição da fauna edáfica. O delineamento foi em blocos casualizados, em esquema fatorial $6 \times 4$, sendo seis culturas de cobertura do solo (aveia preta, nabo, ervilhaca, consórcio nabo/aveia preta, consórcio ervilhaca/nabo/aveia preta e pousio) e quatro épocas de coleta $(0,50,100$ e 150 dias após a semeadura), com quatro repetições. Após a coleta da fauna do solo, foram determinados a abundância de indivíduos, número de colêmbolos, riqueza de Margalef, índice de diversidade de Shannon, equabilidade de Pielou e dominância de Simpson. A utilização dos consórcios nabo/aveia preta e ervilhaca/nabo/ aveia preta favoreceu a abundância de indivíduos e o número de colêmbolos, já nos primeiros 50 dias. O consórcio ervilhaca/ nabo/aveia preta reduziu a diversidade de Shannon, por aumentar a população de colêmbolos. A maior diversidade de espécies foi observada aos 100 dias, após a implantação das culturas. A fauna edáfica foi influenciada pela fase vegetativa e pelo tipo de cultura de cobertura do solo.

PALAVRAS-CHAVE: Avena stringosa; Raphanus sativus L.; Vicia sativa; índices ecológicos; bioindicadores.

tem sido comum o uso de culturas de cobertura de inverno, em solos destinados à produção agrícola, no Estado do Rio Grande do Sul.

Em termos biológicos, a fauna do solo pode ser beneficiada pelo aumento na qualidade e na quantidade de resíduos vegetais que servem de alimento e abrigo para estes organismos edáficos (Baretta et al. 2003). Entretanto, sistemas de monoculturas, ao fornecerem um único substrato orgânico, podem provocar perdas de diversidade biológica do

1. Trabalho recebido em out./2012 e aceito para publicação em abr./2013 ( $n^{\circ}$ registro: PAT 20534).

2. Universidade Federal de Santa Maria (UFSM), Campus Frederico Westphalen, Frederico Westphalen, RS, Brasil.

E-mails: rodrigosilva@smail.ufsm.br, geomarmateus@hotmail.com, gilvanbertollo@yahoo.com.br, santi_pratica@yahoo.com.br.

3. Universidade Federal de Santa Maria (UFSM), Centro de Ciências Rurais, Santa Maria, RS, Brasil.

E-mail: bemfica_steffen@yahoo.com.br. 
solo (Baretta et al. 2003). Contudo, pouco se sabe sobre o efeito do cultivo de culturas de cobertura, ou consórcios de culturas, sobre a comunidade da fauna do solo.

Santos et al. (2008) encontraram efeitos significativos de plantas de cobertura sobre os grupos taxonômicos e densidade relativa da macrofauna edáfica e concluíram que plantas de cobertura da família das leguminosas favorecem a maior densidade relativa de invertebrados no solo. Baretta et al. (2006) encontraram menor diversidade da fauna edáfica, em preparo convencional mantido livre de vegetação. Estudos conduzidos por Alves et al. (2006), em Latossolo Vermelho distroférrico do Estado de São Paulo, indicaram que a ausência de preparo do solo, a abundância de cobertura no sistema plantio direto e o maior tempo de implantação proporcionaram maior diversidade da fauna edáfica.

Os organismos da fauna edáfica são capazes, ainda, de modificar as características físicas, químicas e biológicas do solo, constituindo-se em componentes importantes para a avaliação da organização e funcionamento do mesmo (Steffen et al. 2007). A macrofauna do solo, por participar ativamente nas interações que se estabelecem entre os processos químicos, físicos e biológicos, tem sido apontada como um bom indicador da qualidade do solo (Doran \& Zeiss 2000, Lavelle et al. 2006, Silva et al. 2006), devido à sensibilidade a modificações no meio, respondendo com relativa rapidez, comparativamente a outros indicadores de qualidade do solo, tais como propriedades físicas e carbono orgânico (Reichert et al. 2003).

Grupos funcionais da fauna edáfica podem desaparecer, ou serem reduzidos, em sua abundância e diversidade, como resultado de processos de degradação do solo (Lavelle 1996, Loranger et al. 1999). Santos et al. (2008) observaram diferença significativa nas médias populacionais de artrópodes, com a utilização de plantas de cobertura de inverno, em comparação ao pousio. Neste sentido, é possível que as culturas de cobertura de inverno, utilizadas para proteção do solo, afetem significativamente a comunidade da fauna do solo.

Assim, este trabalho objetivou determinar a influência dos cultivos de aveia preta, nabo e ervilhaca e dos consórcios nabo/aveia preta e ervilhaca/ nabo/aveia preta sobre a estrutura e composição da fauna edáfica.

\section{MATERIAL E MÉTODOS}

O experimento foi realizado em área experimental da Universidade Federal de Santa Maria, em Frederico Westphalen (RS) $\left(27^{\circ} 23^{\prime} 45,75^{\prime}\right.$ 'S, $53^{\circ} 25^{\prime} 45,92^{\prime \prime} \mathrm{W}$ e altitude aproximada de 566 metros), de abril a outubro de 2009. O solo do local é classificado como Latossolo Vermelho distrófico típico (Embrapa 2006), coberto com gramíneas nativas e preparado para implantação das culturas de cobertura.

A análise do solo, seguindo-se metodologia da Embrapa (1997), apresentou as seguintes características físicas e químicas: $39 \%$ de argila; $32 \%$ de areia; $29 \%$ de silte; $\mathrm{pH}$ água de 6,$1 ; 4,3 \mathrm{mg} \mathrm{dm}^{-3} \mathrm{de}$ $\mathrm{P}$ (Mehlich-1); $114 \mathrm{mg} \mathrm{dm}^{-3}$ de K; 11,6 $\mathrm{cmol}_{\mathrm{c}} \mathrm{dm}^{-3}$ de $\mathrm{Ca}^{2+} ; 5,0 \mathrm{cmol}_{\mathrm{c}} \mathrm{dm}^{-3}$ de $\mathrm{Mg}^{2+}$; e 3,4\% de matéria orgânica.

A área apresenta relevo suavemente ondulado e o clima da região é do tipo Cfa, segundo Köppen. Durante o ano de condução do trabalho, a temperatura média anual foi de $20,5^{\circ} \mathrm{C}$, com máximas de $38^{\circ} \mathrm{C}$ e mínimas de $-0,6^{\circ} \mathrm{C}$. A precipitação anual foi de $2.074 \mathrm{~mm}$.

$\mathrm{O}$ trabalho constou de um levantamento da fauna edáfica, em parcela de 3,0 m x 10,0 m. O delineamento experimental utilizado foi em blocos casualizados, em fatorial $6 \times 4$, sendo seis manejos de cobertura do solo (aveia preta - Avena stringosa, nabo - Raphanus sativus L. e ervilhaca - Vicia sativa e os consórcios nabo/aveia preta e ervilhaca/nabo/ aveia preta, mais a parcela pousio) e quatro épocas de coleta $(0,50,100$ e 150 dias após a semeadura), com quatro repetições.

A primeira coleta foi realizada em junho de 2009, antes da implantação das culturas, com o solo totalmente revolvido e manejado sob sistema convencional, sendo utilizada como avaliação inicial para todos os cultivos (Tabela 1). A segunda coleta (50 dias) ocorreu em pleno desenvolvimento das culturas, a terceira (100 dias) durante a fase reprodutiva das culturas e a quarta (150 dias) em novembro de 2009 , ao final do ciclo das culturas, quando se apresentavam como cobertura de solo.

As coletas foram realizadas com o auxílio de armadilhas tipo PROVID (Antoniolli et al. 2006), as quais permaneceram no campo por um período de sete dias, apresentando, em seu interior, 200,0 mL de álcool $70 \%$, para conservação dos insetos. Os indivíduos extraídos nas armadilhas foram identificados quanto à classe ou ordem, com o auxílio de lupas binoculares com aumento de 40 vezes. 
Tabela 1. Grupos da fauna do solo e número de indivíduos identificados na área experimental, antes da instalação do experimento, e respectivos valores dos índices de abundância (AB), riqueza de Margalef (IM) e total de grupos (TG), com média de quatro repetições (Frederico Westphalen, RS, 2009).

\begin{tabular}{|c|c|c|c|c|c|c|c|c|}
\hline \multicolumn{6}{|c|}{ Grupos da fauna do solo } & \multicolumn{3}{|c|}{ Índices } \\
\hline Aca & Ara & Cole & Coll & Dip & Hym & $\mathrm{AB}$ & IM & TG \\
\hline \multicolumn{6}{|c|}{ Número de indivíduos } & \multicolumn{3}{|c|}{ Valores } \\
\hline 1 & 1 & 1 & 170 & 5 & 62 & 240 & 1,51 & 6 \\
\hline
\end{tabular}

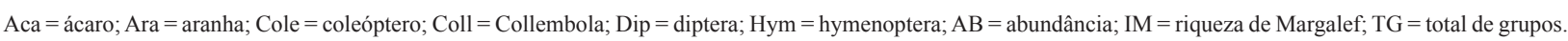

A partir da contagem e identificação dos grupos de organismos edáficos, foram calculados os seguintes índices de biodiversidade: riqueza de organismos; índice de riqueza de Margalef (probabilidade de os indivíduos amostrados em uma área pertencerem a espécies diferentes, com $\mathrm{I}=[(\mathrm{n}-1)] / \mathrm{ln} \mathrm{N}$, onde I é a diversidade, $n$ o número de espécies presente e $\mathrm{N}$ o número total de indivíduos encontrados na amostra); índice de Simpson (forma de dominância dada por $\mathrm{S}=\sum(\mathrm{ni} / \mathrm{N})^{2}$, sendo ni = número de indivíduos do grupo $i \mathrm{e} \mathrm{N}=$ somatório da densidade de todos os grupos) (Odum 1988); índice de diversidade de Shannon $\left(\mathrm{H}=-\sum P i \log P i\right.$, onde $P i$ é a proporção do grupo $i$ no total da amostra) (Shannon \& Weaver 1949). Para a análise da uniformidade da comunidade, foi utilizado o índice de equabilidade de Pielou ( $\mathrm{e}=\mathrm{H} / \mathrm{Log} \mathrm{S}$, onde $\mathrm{H}$ corresponde ao índice de Shannon e $\mathrm{S}$ é o número total de grupos na comunidade) (Pielou 1977).

Os dados de contagem obtidos foram submetidos à transformação $(\mathrm{x}+0,5)^{0,5} \mathrm{e}$ as variáveis submetidas à análise de variância (Anova), utilizando-se o programa Sisvar 5.3 (Ferreira 2008). As médias das variáveis foram comparadas pelo teste Tukey $(\mathrm{p}<0,05)$ e a análise de componentes principais e os índices ecológicos foram determinados pelo software Canoco (Ter Braak \& Smilauer 1998).

\section{RESULTADOS E DISCUSSÃO}

Os resultados das coletas das armadilhas instaladas nas seis áreas avaliadas revelaram diferença de indivíduos da fauna edáfica do solo. Observou-se um total de 1.741 indivíduos da fauna do solo coletados aos 50 dias após o plantio, 1.115 indivíduos aos 100 dias e 1.092 aos 150 dias (Tabela 2). Houve diferença de abundância dos grupos taxonômicos identificados nos tratamentos, durante as diferentes épocas de coleta, sendo que a população de Collembola foi a predominante, em relação aos demais grupos (Tabela 2).
Dentre os grupos encontrados, destacaram-se o Collembola, Acarina e Hymenoptera, os quais representaram $86 \%$ do total de indivíduos (Tabela 2). Estudos envolvendo ácaros (Acarina), colêmbolos (Collembola) e formigas (Hymenoptera), em diferentes agroecossistemas, têm demonstrado grande relevância destes organismos edáficos como indicadores ecológicos de perturbações ambientais, sendo as formigas mais frequentes, especialmente durante o processo de recuperação de áreas degradadas (Andersen et al. 2002, Baretta et al. 2003).

Os colêmbolos pertencem à classe Insecta, subclasse Apterygota, e representam organismos que integram, juntamente com os ácaros, a maior população da mesofauna edáfica (Coleman \& Crossley 1995). A alimentação dos colêmbolos é constituída, principalmente, de fungos ou resíduos vegetais (Coleman \& Crossley 1995, Lavelle 1996) e, também, constituem importante fonte de alimento para outros organismos predadores, como aranhas, coleópteros e ácaros. Além disto, por causa da abundância no solo e sensibilidade a condições ambientais, os colêmbolos são considerados indicadores das condições biológicas do solo (Damé et al. 1996).

Os grupos taxonômicos da fauna do solo encontrados em menor número foram o diptera, araneae, ortóptera e lepidoptera (Tabela 2). Sabe-se que a influência do preparo do solo sobre a frequência da fauna pode ser direta, pelo dano mecânico das operações (Brown et al. 2003), ou, ainda, indireta, pelo aumento da compactação e ausência de cobertura do solo (Baretta et al. 2003, Alves et al. 2006). Deste modo, o preparo inicial realizado na área, antes da instalação do experimento, pode ter favorecido a redução ou repelência de algumas espécies menos adaptadas aos impactos provocados e a diminuição na disponibilidade de alimentos para a fauna edáfica.

$\mathrm{O}$ número de colêmbolos foi influenciado durante a coleta aos 50 dias (Tabela 3), em que os consórcios de ervilhaca/nabo/aveia preta e nabo/aveia 
Tabela 2. Número de indivíduos de cada ordem coletados por parcela $\left(30,0 \mathrm{~m}^{2}\right)$, aos 50, 100 e 150 dias após a semeadura, em função das diferentes culturas e coberturas do solo (Frederico Westphalen, RS, 2009).

\begin{tabular}{|c|c|c|c|c|c|c|}
\hline \multirow{2}{*}{ Classe/Ordem } & \multicolumn{6}{|c|}{ Número de indivíduos em $30,0 \mathrm{~m}^{2}$} \\
\hline & Ap & $\mathrm{Na}$ & Erv & $\mathrm{Na} / \mathrm{Ap}$ & $\mathrm{Ver} / \mathrm{Na} / \mathrm{Ap}$ & Total \\
\hline \multicolumn{7}{|c|}{50 dias } \\
\hline Acarina & 67 & 76 & 22 & 100 & 32 & 297 \\
\hline Araneae & 2 & 0 & 2 & 2 & 2 & 8 \\
\hline Collembola & 56 & 220 & 124 & 307 & 343 & 1.050 \\
\hline Coleoptera & 2 & 3 & 1 & 0 & 1 & 7 \\
\hline Diptera & 29 & 4 & 12 & 4 & 5 & 54 \\
\hline Hemiptera & 0 & 4 & 15 & 3 & 10 & 32 \\
\hline Hymenoptera & 16 & 34 & 24 & 32 & 38 & 144 \\
\hline Larvas & 3 & 0 & 3 & 1 & 1 & 8 \\
\hline Orthoptera & 0 & 0 & 0 & 1 & 0 & 1 \\
\hline Abundância & 175 & 341 & 203 & 450 & 432 & 1.601 \\
\hline Total de grupos & 7 & 6 & 8 & 8 & 8 & \\
\hline \multicolumn{7}{|c|}{100 dias } \\
\hline Acarina & 10 & 15 & 28 & 9 & 7 & 69 \\
\hline Anura & 0 & 0 & 1 & 0 & 0 & 1 \\
\hline Araneae & 11 & 9 & 9 & 4 & 22 & 55 \\
\hline Collembola & 30 & 87 & 63 & 92 & 87 & 359 \\
\hline Coleoptera & 22 & 25 & 13 & 15 & 16 & 91 \\
\hline Diptera & 25 & 29 & 28 & 25 & 30 & 137 \\
\hline Hymenoptea & 41 & 42 & 59 & 30 & 34 & 206 \\
\hline Larvas & 0 & 0 & 2 & 0 & 1 & 3 \\
\hline Orthoptera & 0 & 1 & 0 & 2 & 1 & 4 \\
\hline Abundância & 139 & 208 & 203 & 177 & 198 & 925 \\
\hline Total de grupos & 6 & 7 & 8 & 7 & 8 & \\
\hline \multicolumn{7}{|c|}{150 dias } \\
\hline Acarina & 23 & 13 & 41 & 17 & 15 & 109 \\
\hline Araneae & 2 & 2 & 5 & 5 & 2 & 16 \\
\hline Collembola & 129 & 111 & 187 & 111 & 107 & 645 \\
\hline Coleoptera & 6 & 4 & 4 & 4 & 2 & 20 \\
\hline Diptera & 5 & 0 & 5 & 11 & 2 & 23 \\
\hline Hemiptera & 0 & 0 & 0 & 0 & 0 & 0 \\
\hline Hymenoptera & 12 & 32 & 32 & 27 & 16 & 119 \\
\hline Larvas & 0 & 0 & 0 & 1 & 0 & 1 \\
\hline Lepidoptera & 1 & 0 & 0 & 0 & 1 & 2 \\
\hline Orthoptera & 2 & 2 & 2 & 1 & 2 & 9 \\
\hline Abundância & 180 & 164 & 276 & 177 & 147 & 944 \\
\hline Total de grupos & 8 & 6 & 7 & 8 & 8 & \\
\hline
\end{tabular}

$\mathrm{Ap}=$ aveia preta $\mathrm{Na}=$ nabo Erv = ervilhaca $\mathrm{Na} / \mathrm{Ap}=$ nabo + aveia preta; Ver/ $\mathrm{Na} / \mathrm{Ap}=$ ervilhaca + nabo + aveia preta $;$ Pou $=$ Pousio.

preta apresentaram as maiores populações, com $343 \mathrm{e}$ 307 indivíduos, respectivamente, totalizando $57 \%$ do que foi coletado, enquanto a aveia preta apresentou a menor população de colêmbolos, com 56 indivíduos (Tabela 3). Segundo Lavelle (1996), grupos funcionais da fauna edáfica podem ser substituídos por poucos organismos oportunistas e altamente adaptados a distúrbios, e isto pode ter ocorrido durante a coleta aos 50 dias, quando a população de colêmbolos foi mais abundante.

Aos 100 e 150 dias, com o desenvolvimento das culturas, o número de colêmbolos não apresentou diferença (Tabela 3). Dentre as épocas de coleta, os
Tabela 3. Número de colêmbolos, índice de abundância e riqueza de Margalef, em parcelas de 30,0 $\mathrm{m}^{2}$, aos 50, $100 \mathrm{e}$ 150 dias após a semeadura, em função das diferentes culturas e coberturas do solo (Frederico Westphalen, RS, 2009).

\begin{tabular}{|c|c|c|c|c|}
\hline \multirow{2}{*}{ Cultivos } & \multicolumn{4}{|c|}{ Período (dias) } \\
\hline & $0^{(1)}$ & 50 & 100 & 150 \\
\hline \multicolumn{5}{|c|}{ Colêmbolos } \\
\hline Aveia preta (Ap) & $144 \mathrm{~A}^{(2)}$ & $56 \mathrm{ABc}$ & $30 \mathrm{Ba}$ & $129 \mathrm{Aba}$ \\
\hline Ervilhaca (Er) & $144 \mathrm{~A}$ & $124 \mathrm{Abc}$ & $63 \mathrm{Aa}$ & $187 \mathrm{Aa}$ \\
\hline Nabo $(\mathrm{Nb})$ & $144 \mathrm{~A}$ & $220 \mathrm{Aab}$ & $87 \mathrm{Aa}$ & $111 \mathrm{Aa}$ \\
\hline $\mathrm{Nb} / \mathrm{Ap}$ & $144 \mathrm{AB}$ & $307 \mathrm{Aa}$ & $92 \mathrm{Ba}$ & $111 \mathrm{Ba}$ \\
\hline $\mathrm{Er} / \mathrm{Nb} / \mathrm{Ap}$ & $144 \mathrm{~B}$ & $343 \mathrm{Aa}$ & $87 \mathrm{Ba}$ & $107 \mathrm{Ba}$ \\
\hline Pousio & $144 \mathrm{~A}$ & $75 \mathrm{Abc}$ & $131 \mathrm{Aa}$ & $68 \mathrm{Aa}$ \\
\hline CV (\%) & \multicolumn{4}{|c|}{30} \\
\hline \multicolumn{5}{|c|}{ - Índice de abundância } \\
\hline Aveia preta (Ap) & $214 \mathrm{~A}$ & $175 \mathrm{Abc}$ & $139 \mathrm{Aa}$ & $180 \mathrm{Aa}$ \\
\hline Ervilhaca (Er) & $214 \mathrm{~A}$ & $203 \mathrm{Aa}$ & $203 \mathrm{Aa}$ & $276 \mathrm{Aa}$ \\
\hline Nabo $(\mathrm{Nb})$ & $214 \mathrm{AB}$ & $341 \mathrm{Aab}$ & $208 \mathrm{ABa}$ & $164 \mathrm{Ba}$ \\
\hline $\mathrm{Nb} / \mathrm{Ap}$ & 214 B & $450 \mathrm{Aa}$ & $177 \mathrm{Ba}$ & $177 \mathrm{Ba}$ \\
\hline $\mathrm{Er} / \mathrm{Nb} / \mathrm{Ap}$ & $214 \mathrm{~B}$ & $432 \mathrm{Aa}$ & $198 \mathrm{Ba}$ & $147 \mathrm{Ba}$ \\
\hline Pousio & $214 \mathrm{~A}$ & $140 \mathrm{Ac}$ & $190 \mathrm{Aa}$ & $148 \mathrm{Aa}$ \\
\hline CV (\%) & \multicolumn{4}{|c|}{20} \\
\hline \multicolumn{5}{|c|}{ Riqueza de Margalef } \\
\hline Aveia preta (Ap) & $1,62 \mathrm{Aa}$ & $2,42 \mathrm{Aa}$ & $2,45 \mathrm{Aa}$ & $2,88 \mathrm{Aa}$ \\
\hline Ervilhaca (Er) & $1,62 \mathrm{Ba}$ & $2,76 \mathrm{ABa}$ & $3,18 \mathrm{Aa}$ & $2,47 \mathrm{Aba}$ \\
\hline Nabo $(\mathrm{Nb})$ & $1,62 \mathrm{Aa}$ & $1,87 \mathrm{Aa}$ & $2,39 \mathrm{Aa}$ & $2,41 \mathrm{Aa}$ \\
\hline $\mathrm{Nb} / \mathrm{Ap}$ & $1,62 \mathrm{Aa}$ & $2,27 \mathrm{Aa}$ & $2,59 \mathrm{Aa}$ & $2,79 \mathrm{Aa}$ \\
\hline $\mathrm{Er} / \mathrm{Nb} / \mathrm{Ap}$ & $1,62 \mathrm{Aa}$ & $2,18 \mathrm{Aa}$ & $2,66 \mathrm{Aa}$ & $2,95 \mathrm{Aa}$ \\
\hline Pousio & $1,62 \mathrm{Aa}$ & $2,49 \mathrm{Aa}$ & $2,55 \mathrm{Aa}$ & $1,97 \mathrm{Aa}$ \\
\hline $\mathrm{CV}(\%)$ & \multicolumn{4}{|c|}{15} \\
\hline
\end{tabular}

(1) Valores do tempo zero (0), referente à avaliação da fauna edáfica em toda a área do experimento, antes da instalação dos cultivos. ${ }^{(2)}$ Médias seguidas de mesma letra minúscula, na coluna, e maiúscula, na linha, não diferem entre si, pelo teste Tukey, a 5\%.

consórcios de nabo/aveia preta e ervilhaca/nabo/aveia preta obtiveram os maiores resultados aos 50 dias, enquanto a aveia preta obteve o maior número de colêmbolos no tempo zero (Tabela 3). As demais épocas não diferiram entre si, para os diferentes cultivos.

Conforme Chauvat et al. (2003), a diversidade da estrutura da cobertura vegetal pode influenciar na variabilidade da distribuição de comunidades de colêmbolos edáficos. Loranger et al. (1999) encontraram redução na população e na riqueza de espécies de colêmbolos, em sítios que perderam a cobertura vegetal.

A abundância de indivíduos diferiu aos 50 dias (Tabela 3), sendo observados os melhores resultados nos cultivos consorciados de ervilhaca/nabo/aveia preta e nabo/aveia preta, os quais, juntos, representaram cerca de $50 \%$ do total de indivíduos, enquanto a parcela pousio apresentou a menor abundância, com 140 indivíduos (Tabela 3). Com relação às épocas de 
coleta, os consórcios de ervilhaca/nabo/aveia preta e nabo/aveia preta e nabo foram os que apresentaram diferença para a abundância, sendo observados os maiores resultados aos 50 dias (Tabela 3 ).

Os resultados de abundância podem estar relacionados à funcionalidade da cobertura vegetal, em relação à quantidade e diversidade da biota edáfica (Jandl et al. 2003). Neste caso, cultivos consorciados em curto prazo parecem ter maior capacidade para contribuir com o restabelecimento da fauna edáfica, quando comparados a cultivos solteiros ou áreas sem cobertura. Sabe-se que a presença de palha na superfície e do dossel vegetativo podem contribuir para a manutenção das taxas de infiltração de água e o acúmulo de matéria orgânica (Agostinetto et al. 2000) e, assim, favorecer, diretamente, a fauna do solo.

$\mathrm{O}$ índice de riqueza de Margalef, por sua vez, não foi influenciado pelos cultivos. Contudo, para as épocas de coleta, aos 100 dias, foi observada maior riqueza para o cultivo de ervilhaca (Tabela 3 ).

$\mathrm{O}$ índice de Pielou foi maior na aveia preta, aos 50 e aos 100 dias, superando os demais cultivos e a parcela pousio (Tabela 4). Observou-se que, para os cultivos de aveia preta, nabo, nabo/aveia preta e ervilhaca/nabo/aveia preta, a coleta aos 100 dias foi a que apresentou a maior uniformidade das comunidades da fauna do solo (Tabela 4). Isto significa que, aos 100 dias, houve uma recomposição da fauna do solo. Tais resultados podem ser justificados pela maior diversidade encontrada neste período (Tabela 4).

Para a variável dominância, houve efeitos aos 50 dias, quando o consórcio de ervilhaca/nabo/aveia preta apresentou os maiores índices. Estes resultados se devem à presença da ordem Collembola, a qual representou cerca de $80 \%$ do total de indivíduos encontrados neste tratamento (Tabela 2). A ordem pode ter sido favorecida pela presença mais diversificada de resíduo vegetal, que constitui uma de suas principais fontes de alimento (Coleman \& Crossley 1995). Devido à sua posição na cadeia trófica, seu papel funcional, nos processos que envolvem a dinâmica da matéria orgânica e sua sensibilidade de resposta a perturbações, a ordem Collembola pode ser considerada importante componente para a qualidade do solo (Coleman \& Hendrix 2000). Tais aspectos ganham relevância, quando se observa, aos 50 dias, um menor número de colêmbolos nos tratamentos solteiros, se comparados a tratamentos consorciados, como é o caso do nabo/aveia preta e ervilhaca/nabo/ aveia preta (Tabela 2).
Tabela 4. Índice de Pielou, dominância e diversidade de Shannon, em parcelas de $30,0 \mathrm{~m}^{2}$, aos 50, 100 e 150 dias após a semeadura, em função das diferentes culturas e coberturas do solo (Frederico Westphalen, RS, 2009).

\begin{tabular}{lllll}
\hline \multirow{2}{*}{ Cultivos } & \multicolumn{5}{c}{$0^{(1)}$ Período (dias) } \\
\cline { 2 - 5 } Indice de Pielou \\
\hline Aveia preta (Ap) & $0.537 \mathrm{~B}^{(2)}$ & $0.722 \mathrm{ABa}$ & $0.872 \mathrm{Aa}$ & $0.515 \mathrm{Ba}$ \\
Ervilhaca (Er) & $0.537 \mathrm{~A}$ & $0.615 \mathrm{Aab}$ & $0.757 \mathrm{Aab}$ & $0.527 \mathrm{Aa}$ \\
Nabo (Nb) & $0.537 \mathrm{~B}$ & $0.542 \mathrm{ABab}$ & $0.802 \mathrm{Aab}$ & $0.535 \mathrm{Ba}$ \\
$\mathrm{Nb} / \mathrm{Ap}$ & $0.537 \mathrm{AB}$ & $0.455 \mathrm{Bab}$ & $0.742 \mathrm{Aab}$ & $0.580 \mathrm{ABa}$ \\
Er/Nb/Ap & $0.537 \mathrm{AB}$ & $0.395 \mathrm{Bb}$ & $0.772 \mathrm{Aab}$ & $0.575 \mathrm{ABa}$ \\
Pousio & $0.537 \mathrm{~A}$ & $0.685 \mathrm{Aab}$ & $0.560 \mathrm{Ab}$ & $0.760 \mathrm{Aa}$ \\
\hline $\mathrm{CV}(\%)$ & \multicolumn{5}{c}{6,4} \\
\hline & \multicolumn{5}{c}{ Indice de dominancia } \\
\hline Aveia preta (Ap) & $0.547 \mathrm{~A}$ & $0.330 \mathrm{ABb}$ & $0.240 \mathrm{Ba}$ & $0.537 \mathrm{Aa}$ \\
Ervilhaca (Er) & $0.547 \mathrm{~A}$ & $0.425 \mathrm{ABab}$ & $0.252 \mathrm{Ba}$ & $0.500 \mathrm{ABa}$ \\
$\mathrm{Nabo}(\mathrm{Nb})$ & $0.547 \mathrm{~A}$ & $0.482 \mathrm{ABab}$ & $0.275 \mathrm{Ba}$ & $0.512 \mathrm{ABa}$ \\
$\mathrm{Nb} / \mathrm{Ap}$ & $0.547 \mathrm{~A}$ & $0.537 \mathrm{Aab}$ & $0.330 \mathrm{Aa}$ & $0.460 \mathrm{Aa}$ \\
Er/Nb/Ap & $0.547 \mathrm{~A}$ & $0.657 \mathrm{Aa}$ & $0.240 \mathrm{Ba}$ & $0.487 \mathrm{ABa}$ \\
Pousio & $0.547 \mathrm{~A}$ & $0.372 \mathrm{Aab}$ & $0.485 \mathrm{Aa}$ & $0.375 \mathrm{Aa}$ \\
\hline $\mathrm{CV}(\%)$ & \multicolumn{5}{c}{7,7} \\
\hline Aveia preta (Ap) & $0.332 \mathrm{~B}$ & $0.572 \mathrm{ABa}$ & $0.695 \mathrm{Aa}$ & $0.447 \mathrm{ABa}$ \\
Ervilhaca (Er) & $0.332 \mathrm{~B}$ & $0.532 \mathrm{ABa}$ & $0.690 \mathrm{Aa}$ & $0.437 \mathrm{ABa}$ \\
$\mathrm{Nabo}(\mathrm{Nb})$ & $0.332 \mathrm{~B}$ & $0.420 \mathrm{ABa}$ & $0.645 \mathrm{Aa}$ & $0.427 \mathrm{ABa}$ \\
$\mathrm{Nb} / \mathrm{Ap}$ & $0.332 \mathrm{~B}$ & $0.380 \mathrm{ABa}$ & $0.610 \mathrm{Aa}$ & $0.497 \mathrm{ABa}$ \\
Er/Nb/Ap & $0.332 \mathrm{~B}$ & $0.315 \mathrm{Ba}$ & $0.632 \mathrm{Aa}$ & $0.477 \mathrm{ABa}$ \\
Pousio & $0.332 \mathrm{~A}$ & $0.537 \mathrm{Aa}$ & $0.460 \mathrm{Aa}$ & $0.520 \mathrm{Aa}$ \\
\hline $\mathrm{CV}(\%)$ & \multicolumn{5}{c}{7,5} \\
\hline
\end{tabular}

(1) Valores do tempo zero (0), referente à avaliação da fauna edáfica em toda a área do experimento, antes da instalação dos cultivos. ${ }^{(2)}$ Médias seguidas de mesma letra minúscula, na coluna, e maiúscula, na linha, não diferem entre si, pelo teste Tukey, a $5 \%$.

O índice de diversidade de Shannon foi influenciado pelo tempo, sendo que, aos 100 dias, a diversidade de espécies foi superior à das demais avaliações (Tabela 4), exceto para o tratamento pousio. Estes resultados se devem, possivelmente, à maior cobertura vegetal oferecida pelos cultivos aos 100 dias. Martins \& Rosa Júnior (2005) e Silva et al. (2006), estudando o cultivo de ervilhaca peluda, relataram quantidades de massa seca acima de $3.000 \mathrm{~kg} \mathrm{ha}^{-1}$, aos 113 dias após a semeadura.

Os resíduos vegetais constituem a principal fonte de alimento e habitat para grande parte das espécies de invertebrados que habitam o solo (Silva et al. 2006). Conforme Lavelle et al. (1992) e Lavelle (1996), a diversidade da fauna edáfica está relacionada com a grande variedade de condições que o sistema solo-serapilheira oferece. Entretanto, neste mesmo período, não foi observada diferença entre os cultivos de cobertura do solo (Tabela 4).

Sabe-se que, quanto mais diversa for a cobertura vegetal, maior será a heterogeneidade da serapilheira, que apresentará maior diversidade das 
comunidades de fauna (Correia \& Andrade 1999). Entretanto, não foram observados efeitos para o índice de diversidade de Shannon entre os cultivos, para as diferentes épocas de coleta (Tabela 4). O que se observou foi um baixo índice de diversidade para o tratamento ervilhaca/nabo/aveia preta, aos 50 dias (Tabela 4). Tais resultados são justificados pela presença de um grande número de indivíduos da ordem dos colêmbolos, considerados indicadores de qualidade do solo, devido à sua flutuação populacional (Sautter \& Santos 1991). A maior abundância desta ordem resultou em aumento na dominância e, portanto, declínio na diversidade, neste período. Contudo, aos 100 dias, a diversidade, para este tratamento, já havia se restabelecido a valores mais elevados (Tabela 4).

Segundo Lavelle (1996), um ganho em diversidade de invertebrados edáficos resulta na produção de estruturas fundamentais para a conservação e dinâmica da matéria orgânica do solo, ciclagem de nutrientes e manutenção de propriedades físicas essenciais para a sustentabilidade da produção primária. Portanto, áreas com baixa presença de organismos edáficos, como ocorre em solos perturbados, desprotegidos e com pouca cobertura de solo, são prejudiciais à manutenção do equilíbrio e da qualidade do solo.

De acordo com a análise multivariada dos dados obtidos neste trabalho (Figura 1), observou-se variação quanto à eficiência dos tratamentos. Ao longo da dimensão 1 (eixo principal), os tratamentos ervilhaca/nabo/aveia preta e pousio estão dispostos na porção mais extrema à direita (com valores positivos) e os tratamentos nabo/aveia preta na porção mediana, enquanto os tratamentos com nabo, aveia preta e ervilhaca estão dispostos na porção esquerda (com valores negativos), demonstrando separação entre os cultivos.

O índice de abundância apresentou maior relação com o tratamento pousio e, em escala menor, com o consórcio ervilhaca/nabo/aveia preta. Para a dominância de Simpson e número de colêmbolos, estes apresentaram associação com os cultivos de nabo, aveia preta e ervilhaca, porém, com pouca contribuição, devido ao fato de estes cultivos se encontrarem na parte mediana dos eixos. O mesmo aconteceu para o índice de Shannon, segundo o qual o consórcio nabo/aveia preta foi o que apresentou maior associação, apesar de o mesmo se encontrar na parte mediana do eixo.

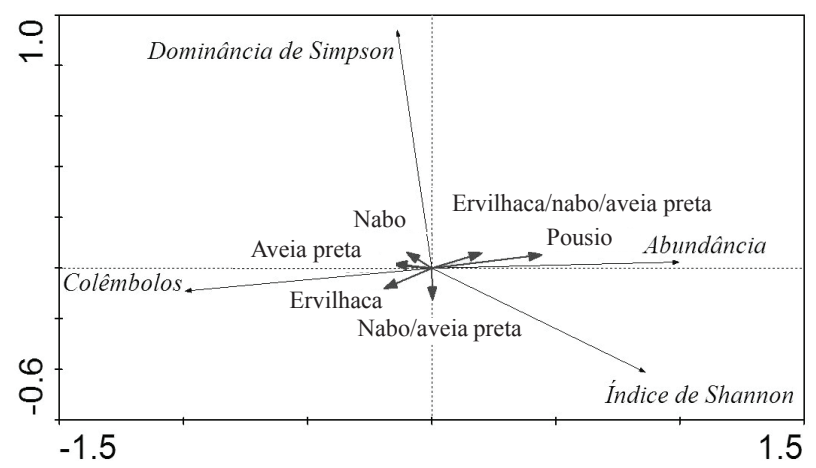

Figura 1. Representação gráfica da análise de componentes principais (PCA), relacionando as dimensões 1 e 2 , referentes à abundância da fauna do solo, índice de dominância de Simpson, índice de diversidade de Shannon e número de colêmbolos, sob a influência de culturas de cobertura: nabo, aveia preta, ervilhaca, pousio e os consórcios nabo/aveia preta e ervilhaca/nabo/aveia preta (Frederico Westphalen, RS, 2009).

Segundo Moço et al. (2005), a maior ou menor associação de um determinado grupo da fauna edáfica, em cada tratamento, deve-se ao tipo de preparo do solo e, principalmente, aos efeitos benéficos dos resíduos vegetais mantidos na superfície do solo, que proporcionam ambiente mais favorável à sobrevivência de determinados grupos. De acordo com Baretta et al. (2006), a fauna do solo pode ser utilizada como bioindicador das alterações advindas do manejo do solo, especialmente pelo emprego de ferramentas estatísticas multivariadas.

\section{CONCLUSÕES}

1. A utilização de consórcios entre culturas de cobertura favoreceu o aumento da abundância de indivíduos e do número de colêmbolos, nas fases iniciais.

2. Os organismos da fauna edáfica apresentaram comportamento diferenciado, em função do tipo e do período de desenvolvimento das culturas de cobertura.

\section{REFERÊNCIAS}

AGOSTINETTO, D. et al. Adaptação de espécies utilizadas para cobertura de solo no sul do Rio Grande do Sul. Revista Brasileira de Agrociência, Pelotas, v. 6, n. 1, p. 47-52, 2000. 
ALCÂNTARA, F. A. et al. Adubação verde na recuperação da fertilidade de um Latossolo Vermelho-escuro degradado. Pesquisa Agropecuária Brasileira, Brasília, DF, v. 35, n. 2, p. 277-288, 2000.

ALVES, M. V.; BARETTA, D.; CARDOSO, E. J. B. N. Fauna edáfica em diferentes sistemas de cultivo no Estado de São Paulo. Revista de Ciências Agroveterinárias, Lages, v. 5, n. 1, p. 33-43, 2006.

ANDERSEN, A.; BENJAMIN, D. H.; MÜLLER, $\mathrm{W}$. Using ants as bioindicators in land management: simplifying assessment of ant community responses. Journal of Applied Ecology, Oxford, v. 39, n. 1, p. 8-17, 2002.

ANTONIOLLI, Z. I. et al. Método alternativo para estudar a fauna do solo. Ciência Florestal, Santa Maria, v. 16, n. 4, p. 407-417, 2006.

BARETTA, D. et al. Análise multivariada da fauna edáfica em diferentes sistemas de preparo e cultivo do solo. Pesquisa Agropecuária Brasileira, Brasília, DF, v. 41, n. 11, p. 1675-1679, 2006.

BARETTA, D. et al. Fauna edáfica avaliada por armadilhas de catação manual afetada pelo manejo do solo na região oeste catarinense. Revista Ciência Agroveterinária, Lages, v. 2, n. 1, p. 97-106, 2003.

BOER, C. A. et al. Ciclagem de nutrientes por plantas de cobertura na entressafra em um solo de Cerrado. Pesquisa Agropecuária Brasileira, Brasília, DF, v. 42, n. 9, p. 12691276, 2007.

BROWN, G. G. et al. No-tillage greatly increases earthworm populations in Paraná State, Brasil. Pedobiologia, Jena, v. 47, n. 5-6, p. 764-771, 2003.

CHAUVAT, M.; ZAITSEV, A. S.; WOLTERS, V. Successional changes of Collembola and soil microbiota during forest rotation. Oecologia, Berlin, v. 137, n. 2, p. 269-276, 2003.

COLEMAN, D. C.; CROSSleY JUNIOR, D. A. Fundamentals of soil ecology. San Diego: Elsevier, 1995.

COLEMAN, D. C.; HENDRIX, P. F. Invertebrates as webmasters in ecosystems. London: CABI Publishing, 2000.

CORREIA, M. E. F.; ANDRADE, A. G. Formação de serapilheira e ciclagem de nutrientes. In: SANTOS, G. A.; CAMARGO, F. A. O. (Eds.). Fundamentos da matéria orgânica do solo: ecossistemas tropicais e subtropicais. Porto Alegre: Gênesis, 1999. p. 197-225.

COSTA, M. S. S. M. et al. Nutrição e produtividade da cultura do milho em sistemas de culturas e fontes de adubação. Revista Ceres, Viçosa, v. 58, n. 2, p. 249-255, 2011.
DAMÉ, P. R. V. et al. Efeitos da queimada seguida de pastoreio ou diferimento sobre o resíduo, temperatura do solo e mesofauna de uma pastagem natural. Ciência Rural, Santa Maria, v. 26, n. 3, p. 391-396, 1996.

DORAN, J. W.; ZEISS, M. R. Soil health and sustainability: managing the biotic vompont of soil quality. Applied Soil Ecology, Pretty, v. 15, n. 1, p. 3-11, 2000.

EMPRESA BRASILEIRA DE PESQUISA AGROPECUÁRIA (Embrapa). Centro Nacional de Pesquisa de Solos. Manual de métodos de análises de solo. 2. ed. Rio de Janeiro: Embrapa-CNPS, 1997.

EMPRESA BRASILEIRA DE PESQUISA AGROPECUÁRIA (Embrapa). Sistema brasileiro de classificação de solos. 2. ed. Rio de Janeiro: EmbrapaCNPS, 2006.

FERREIRA, D. F. Sisvar: um programa para análises e ensino de estatística. Revista Symposium, Lavras, v. 6 , n. 1, p. 36-41, 2008.

JANDL, R. et al. Forest soil chemistry and mesofauna 20 years after an amelioration fertilization. Restoration Ecology, Malden, v. 11, n. 2, p. 239-246, 2003.

LAVELLE, P. Diversity of soil fauna and ecosystem function. Biology Internship, Paris, v. 33, n. 1, p. 3-16, 1996.

LAVELLE, P. et al. Impact of soil fauna on the properties of soils in the humid tropics. Madison: SSSA, 1992. (Special publication, 29).

LAVELLE, P. et al. Soil invertebrates and ecosystem services. European Journal of Soil Biology, Jersey, v. 42, n. 1, p. 3-15, 2006.

LORANGER, G. et al. Influence of agricultural practices on arthropod communities in a vertisol (Martinique). European Journal of Soil Biology, Jersey, v. 34, n. 3, p. 157-165, 1999.

MARTINS, R. M. G.; ROSA JÚNIOR, E. J. Culturas antecessoras influenciando a cultura de milho e os atributos do solo no sistema de plantio direto. Acta Scientiarum Agronomy, Maringá, v. 27, n. 2, p. 225-232, 2005.

MOÇO, M. K. et al. Caracterização da fauna edáfica em diferentes coberturas vegetais na região norte fluminense. Revista Brasileira de Ciência do Solo, Viçosa, v. 29, n. 4, p. 555-564, 2005.

ODUM, E. P. Ecologia. Rio de Janeiro: Guanabara Koogan, 1988.

PIELOU, E. C. Mathematical ecology. New York: Wiley, 1977.

REICHERT, J. M.; REINERT, D. J.; BRAIDA, J. A. Qualidade do solo e sustentabilidade de sistemas agrícolas. 
Ciência \& Ambiente, Santa Maria, v. 27, n. 1, p. 29-48, 2003.

SANTOS, G. G. et al. Macrofauna edáfica associada a plantas de cobertura em plantio direto em um Latossolo Vermelho do Cerrado. Pesquisa Agropecuária Brasileira, Brasília, DF, v. 43, n. 1, p. 115-122, 2008.

SAUTTER, K. D.; SANTOS, H. R. Insetos bioindicadores na recuperação de solos. Ciência Hoje, Belo Horizonte, v. 12, n. 72, p. 20-21, 1991.

SHANNON, E. V.; WEAVER, W. The mathematical theory of communication. Urbana: University of Illinois Press, 1949.
SILVA, R. F. et al. Macrofauna invertebrada do solo sob diferentes sistemas de produção em latossolo da região do Cerrado. Pesquisa Agropecuária Brasileira, Brasília, DF, v. 41, n. 4, p. 697-704, 2006.

STEFFEN, R. B.; ANTONIOLLI, Z. I.; STEFFEN, G. P. K. Avaliação de substratos para reprodução de colêmbolos nativos em condições de laboratório. Ciência Florestal, Santa Maria, v. 17, n. 3, p. 265-269, 2007.

TER BRAAK, C. J. F.; SMILAUER, P. C. Reference manual and user's guide to Canoco for Windows: software for canonical community ordination. Version 4.0. New York: Microcomputer Power, 1998. 